\title{
Chiral Solid Solutions for the NMR Analysis of Enantiomers: A Potential New Approach to Chiral Analysis
}

\author{
Karel D. Klika \\ Department of Chemistry, University of Turku, Vatselankatu 2, 20014 Turku, Finland \\ Correspondence should be addressed to Karel D. Klika; klikakd@yahoo.co.uk
}

Received 10 June 2012; Revised 10 August 2012; Accepted 13 August 2012

Academic Editor: Eugen Culea

Copyright (C) 2013 Karel D. Klika. This is an open access article distributed under the Creative Commons Attribution License, which permits unrestricted use, distribution, and reproduction in any medium, provided the original work is properly cited.

Differences between the solid-state ${ }^{13} \mathrm{C}$ CP-MAS NMR spectra of holemic samples of the two enantiomers of $2,2^{\prime}$-dihydroxy-1,1'binaphthyl (binol) were not sufficiently emphatic to reliably distinguish them, though they are readily distinguishable from the spectrum of the bimate of the compound crystallized from an equimatic sample. Inducing an additional chiral environment by cocondensation with sucrose as a chiral selector (CS) provided a method to yield differential spectra for the two enantiomers and thus effect enantiodifferentiation by way of solid-state NMR using weak interactions from a CS.

\section{Introduction}

One of the most important and challenging determinations in chemistry is the state of chirality ("enantiodifferentiation," i.e., the identification of an enantiomer, the quantification of enantiomeric composition, and/or the determination of absolute configuration $[1,2]$ ), ever more so with the increasingly stringent requirements placed upon the pharmaceutical industry for enantiomerically pure drugs. As part of our investigations [3] into chiral processes involving weak interactions, in particular the ESDAC [3-7] (enantiomer self-disproportionation on achiral chromatography) phenomenon stemming from our original observations $[4,8]$ of 9-hydroxy cineole (1) and its related metabolites (Figure 1) such as the dihydroxylated compounds 2,9- (2) and 2,10dihydroxy-1,8-cineole (3) [9], we were greatly interested in new methods of enantiodifferentiation. 9-Hydroxy cineole (1) is an interesting compound because it readily undergoes SDE (self-disproportionation of enantiomers) [10], for example, by ESDAC [4] and also by sublimation [8], since it, like $\mathbf{2}$ and $\mathbf{3}$, is obtained as a scalemate from its natural source. A scalemate is a sample containing an unequal mixture of enantiomers-the preferred terminology and descriptors set out recently $[11,12]$ with respect to chiral systems are used herein. Thus, not only were we interested in absolute configuration determination but also quantification of the enantiomeric composition (ec), and for which we had to take care not to elicit perturbation of the ec from its original state.

Fortunately, however, many methods are available to accomplish enantiodifferentiation and even within NMR there are a number of broad approaches [13-19] including chemical derivatization, chiral solvating agents (CSAs), and chiral complexation. In addition, chiral cavitands, either under isotropic conditions [17, 20-24] or otherwise [25], have also been explored with respect to evaluation of the ec and/or identification of the predominant enantiomer. The most formidable advances within NMR of recent times in this context have involved the application of chiral liquid crystals (CLCs) [26-38] —originating from the pioneering work [39, 40] of Snyder et al.-for determination of the ec or even for absolute configuration determination. Such are the dramatic differences in favorable cases that even (fluoro)hydrocarbon enantiomers [31-33] and enantiomers chiral by virtue of isotopic substitution [36] have been successfully analyzed using this methodology. The great success of CLCs lies in the strong response by various order-dependent parameters to the small differences between the enantiomers in the chiral environment based on their orientation to the field; though differential orientation of enantiomers by other, fieldindependent media is also possible [41, 42]. However, despite all efforts, a general solution $[13,19,27,28,43]$ to the central questions has not been found. A general solution is not only 


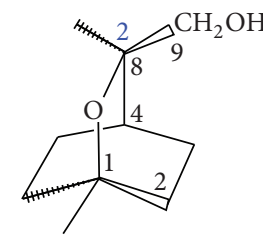

1

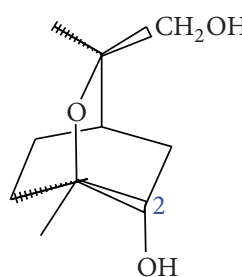

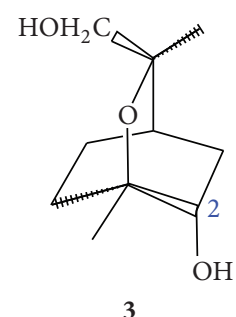

3
FIgURE 1: The structures of compounds 1-3. The recently described [11] notation for indicating relative stereochemistry as an extension to the Natta projection convention proposed by Giulio Natta (1903-1979) is used and for which the " 2 " near to the C-2 and C8 atoms indicates that the number of stereoisomers present in the sample or under consideration is two.

desirable in its own right but also to limit the shortcomings of the various present methodologies [13-15, 28, 41, 42] which include technical and interpretative challenges. All of the previous work, however, suggests that if a general solution is to be found, then the solution will likely involve a compact state and/or aligned media. In this work, the aim is to examine a new approach to chiral analysis in the solid state by use of a chiral selector (CS), namely, sucrose, to effect the enantiodifferentiation of 2,2' -dihydroxy-1,1' - binaphthyl (4, binol) by means of ${ }^{13} \mathrm{C}$ CP-MAS NMR (cross-polarizationmagic angle spinning NMR).

\section{Results and Discussion}

One intriguing notion is whether enantiomers are able to display observable spectroscopic differences under isotropic conditions (in addition to parity violation effects [44]): in dilute solution (considering the typical concentrations used for solution-state NMR), the presumption is that the analyte molecules are well separated and effectively prevented from intimate interaction with each other by solvation. This is obviously not the case in the solid state but, thus far at least, there are no reports of such observations. Applying the same rationalization for an isolated pair of chiral molecules (considering them equivalent in energy) to a chiral molecule in a matrix of identical molecules, then the presumption would be that this system is equivalent to its mirror-image molecule also surrounded by identical molecules. Distinctions between holemates (i.e., enantiopure samples) and their equimates (i.e., samples consisting of $1: 1$ ratios of the two enantiomers) have been reported in nonisotropic phases [13, 18, 45-47], and distinctions have even been made on the order-dependent parameters for cases where the holmate and the bimate (i.e., the "racemate") exhibit identical isotropic chemical shifts and anisotropic values $[48,49]$. These differences can even permit quantitation of the ec for scalemates in favorable cases in either of the aforementioned situations. However, these observations are attributed to the different space groups that these particular enantiomers and their corresponding equimates belong to $[50,51]$ in the former case, or in the latter case, due to the differing molecular symmetries and packings for the

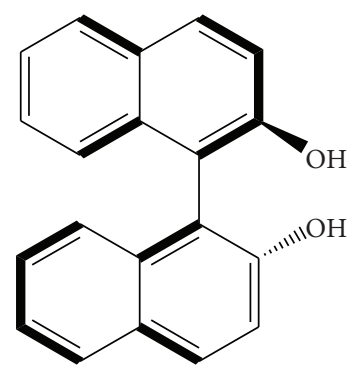

$R-(+)-\operatorname{binol}(R-4)$

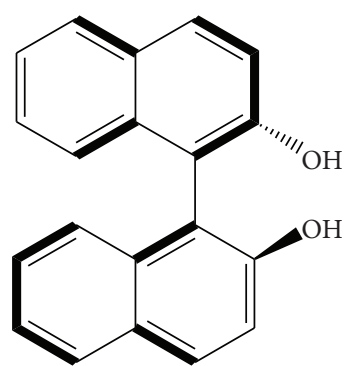

S-(-)-binol (S-4)

Figure 2: The absolute configurations of the two enantiomers, $R-4$ and $S-4$, of $2,2^{\prime}$-dihydroxy-1, $1^{\prime}$-binaphthyl (4, binol).

molecules in the holematic and bimatic states [48, 49]. These results have been suitably reviewed [50,51]. Given the lack of differential observations between the spectra of two enantiomers in the confines of the solid state leads either to the conclusion that there is no discernible effect or that the same net effect is resultant for both enantiomers-and also the equimate in cases where the space groups are the same or the compound behaves as a homomate (conglomerate) or unimate (pseudoracemate). Alternatively, perturbation of the chiral environment in the solid state by the introduction of a CS could also lead to enantiodifferentiation. This approach does not seem to have been considered previously, though NMR examination of the enclathration in the solid phase of an enantiomer by a chiral medium has been reported [52] and reviewed [51]. Thus, whilst noncovalent interactions for the creation of a chiral environment have been useful for the analysis of enantiomers [13-19, 28], for example, CSAs have been in use for some time now and have been subsequently followed by CLCs [51], the next logical step is chiral solid solutions (CSSs). Of note, in a weak chiral environment (induced by itself nonetheless), 1 readily displays chiralstate dependent differential behavior and this led us to the premise that perhaps not just in solution-state NMR, but also in solid-state NMR similar phenomena could be observed based on the analogous phenomena of SIDA (self-induced diastereomeric anisochronism) $[3,53]$ in the former method. Herein, enantiodifferentiation by an extremely weak external CS, namely, sucrose, as the provider of a chiral environment, is examined.

To explore the aforementioned premise, binol (4) was examined by solid-state ${ }^{13} \mathrm{C}$ CP-MAS NMR. The two enantiomers of binol ( $R-\mathbf{4}$ and $S \mathbf{- 4}$, see Figure 2$)$ each crystallizes in the $P 3_{2}$ space group whilst the bimate (i.e., a "racemic compound," bim-4) crystallizes in the Iba2 space group [54]. Four samples consisting of holemic samples of each of the two enantiomers ( $R-\mathbf{4}$ and $S-\mathbf{4})$, an equimate sample as a mechanical mixture of the two $(R-4+S-4)$ prepared by the addition and thorough solid-phase mixing of equal amounts of $R-4$ and S-4 using a vortex mixer for 10-15 minutes, and an equimate sample prepared as the bimate (bim-4) by crystallization of an equimatic sample from chloroform 


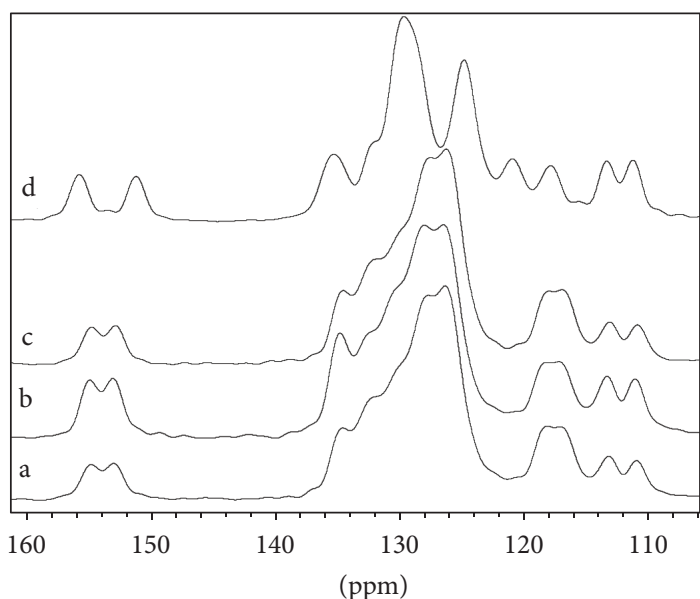

Figure 3: The ${ }^{13} \mathrm{C}$ solid-state NMR spectra of binol (4): $R-\mathbf{4}+S-\mathbf{4}$ (trace a), $R-4$ (trace b), $S-4$ (trace c), and bim-4 (trace d).

were examined. Although solid-phase admixture of the enantiomers $R-\mathbf{4}$ and $S-\mathbf{4}$ has been reported [54] to rapidly yield the bimate, morphing was not apparent in this study upon mixing and the bimate was only formed after dissolution and removal of the solvent by evaporation. The results are portrayed in Figure 3 where the spectrum, as expected, of the bimate (bim-4) is clearly distinct from the spectra of the two enantiomers $R-\mathbf{4}$ and $S-\mathbf{4}$ and the mechanical mixture of the two $(R-\mathbf{4}+S-\mathbf{4})$. However, although there are slight discernible differences between each of the enantiomers and the mechanical mixture in the figure shown, for example, the intensity of the pairs of signals at ca. 112 and $154 \mathrm{ppm}$ and the signal at ca. $134 \mathrm{ppm}$, these apparent distinctions are blurred by the run-to-run inconsistencies of the acquired spectra and thus cannot by unequivocally attributed to the inherent differences between the two enantiomers $R-\mathbf{4}$ and $S-\mathbf{4}$ and the mechanical mixture $R-\mathbf{4}+S-\mathbf{4}$. To confirm that the differences were not due to sample inconsistencies, the samples were also examined by solution-state NMR, whereby the spectra of $R-\mathbf{4}$ and $S-\mathbf{4}$ were found to be, to all intents and purposes, identical by ${ }^{1} \mathrm{H}$ and ${ }^{13} \mathrm{C}$ NMR.

To perturb the intrinsic chiral environment and thereby create discernible differences, an enhanced chiral environment was induced in the samples by the admixture of a CS, and for this purpose sucrose was used. Physical intimacy between the hydrophobic and hydrophilic compounds was ensured by the use of finely powdered samples; in the case of sucrose, consumer-grade crystals were ground in a mortar and pestle prior to admixture whilst the binol (4) was used as received. Following weighing and admixture, thorough dispersion and intimate contact between the crystals was ensured by several minutes of vortex mixing. Several samples were again prepared: the two enantiomers $(R-\mathbf{4}$ and $S-\mathbf{4}$, each ca. 1:1 with the CS and two further samples of $R$ 4 in a 1.19:1 ratio and a $0.55: 1$ ratio), the equimate as a mechanical mixture $(R-\mathbf{4}+S-\mathbf{4}$, ca. $1: 1$ with the $C S)$, and the bimate (bim-4, ca. $1: 1$ with the CS). Again, although in particular comparisons discernible differences were apparent

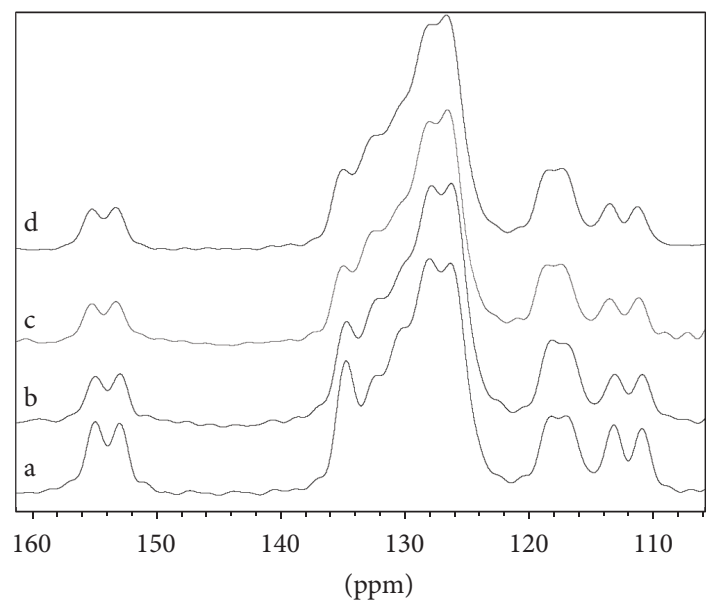

FIgURE 4: The ${ }^{13} \mathrm{C}$ solid-state NMR spectra of the $S$-enantiomer of binol (S-4): an aged sample after cocondensation with sucrose from DSMO solution (trace a), a freshly dried sample after cocondensation (trace b), a physically mixed-only sample of $S$-enantiomer $(S-4)$ and sucrose, ca. $1: 1$ (trace c), and the pure $S$-enantiomer $(S-4)$ (trace d).

in the ${ }^{13} \mathrm{C}$ solid-state NMR, the run-to-run inconsistencies of the acquired spectra again prevented any firm conviction of a distinction between either of the enantiomers $(R-4$ and $S$-4) and the mechanical mixture of the two $(R-4+$ $S$-4). Indeed, for all directly comparable spectra, reliable distinctions between the samples consisting solely of $4(R-$ 4, $S-\mathbf{4}, R-\mathbf{4}+S-\mathbf{4}$, and bim-4) and the admixture of each sample with sucrose were not forthcoming. Needless to say, variation of the enantiomer to sugar ratio failed to realize any significant perturbation.

To accentuate the perturbations of the chiral environment, each of the binol (4) admixtures with sucrose was taken up in DMSO and the resulting solutions then reduced to dryness under high vacuum. These cocondensed samples were then subsequently again examined by ${ }^{13} \mathrm{C} C \mathrm{CP}-\mathrm{MAS}$ NMR. For the $R$ enantiomer $(R-4)$ with sucrose, new distinctions were not apparent above that of the run-to-run inconsistencies. However, most intriguingly, upon close examination of the $S$-enantiomer (S-4) with sucrose, the spectrum of the dried sample was distinct, but this was only evident after the course of several weeks (Figure 4). The main distinctions were for the pairs of signals at ca. $112 \mathrm{ppm}$ and ca. $154 \mathrm{ppm}$ wherein both pairs were substantially increased in intensity, similarly also for the signal at $134 \mathrm{ppm}$. The two peaks at $127 \mathrm{ppm}$ were altered such that a reversal of signal intensity relative between the two occurred. The freshly dried sample also showed these distinctions, but the perturbations were only very slight and were intermediate between the physically mixed-only sample of $S$-enantiomer $(S-4)$ with sucrose and the cocondensed aged sample. It is of considerable note that the differences displayed by the aged $S$-enantiomer $(S-\mathbf{4})$ with sucrose sample appear to exceed the run-to-run inconsistencies and that these distinctions are thus attributable to the induced chiral environment provided by the CS. In principle, therefore, this method could provide a means for enantiomer identification 
or even determination of ec with appropriate data processing (e.g., deconvolution of signals). It must be noted though that cocondensation will form the bimate if the $R$-enantiomer $(R$ 4) is present, so cocondensation is limited to homomates and unimates and not applicable to systems that display heteromatic behavior such as bimates-as is the case here. Moreover, this time-dependent change in the spectra was not apparent for the physically mixed-only sample of the $S$ enantiomer $(S-4)$ with sucrose. Also, clearly distinct changes were not evident over the same course of time for any of the $R$-enantiomer $(R-4)$ with sucrose samples. And finally, differences in the spectra of the CS sucrose were not in evidence.

It should be noted that although CP is not conducive to quantitative studies, in this work, we are not comparing atoms in different molecules nor are we comparing different atoms within the one molecule, but rather the same atoms under different chiral conditions. This has been shown to be a valid assumption [46]. Thus, by maintaining constant $\mathrm{CP}$ conditions across the samples, valid comparisons were ensured.

\section{Conclusions}

Thus, this as yet unexplored avenue of examination could potentially yield a general and easy-to-implement solution for chiral analysis. The obvious appeals of this approach are its simplicity, the ease of sample recovery (in this particular case, only liquid-liquid extraction), the circumvention of specific interactions, and the dispensing of any special technique for sample preparation or spectral interpretation. Thus, whilst the power of CLCs at present is unparalleled, the technical and interpretative challenges limit the chance of a general solution being easily found. With further work it is hoped that development of this approach could be advanced to the stage where enantiomeric identification and the quantification of enantiomeric excess can be accomplished, not only in specific cases, but as a more general solution.

\section{Experimental}

${ }^{13} \mathrm{C}$ CP-MAS NMR spectra were acquired without lock at ambient temperature in a field of $9.4 \mathrm{~T}(100 \mathrm{MHz})$ using $4 \mathrm{~mm} \mathrm{ZrO}_{2}$ rotors spun at $9 \mathrm{kHz}$ with a contact time of $16 \mathrm{~ms}$ and postacquisition delay of $300 \mathrm{~s}$. A long contact time was utilized to facilitate observation of the quaternary carbons via long-range couplings; thus, a compromise value was applied between the one-bond and multiple-bond couplings with this compromise value being determined in practice from a short series of measurements using optimized conditions to locate an appropriately sufficient value. The $90^{\circ}$ pulse for ${ }^{1} \mathrm{H}$ in the irradiation channel $(3.90 \mu \mathrm{s})$ was found by acquisition of a series of ${ }^{13} \mathrm{C} C \mathrm{CP}$-MAS NMR spectra on a standard adamantane sample and locating the optimal value at a set power level. This same power level was used for the Hartman-Hahn condition where CP was effected by continuous wave irradiation of both the ${ }^{1} \mathrm{H}$ and ${ }^{13} \mathrm{C}$ nuclei. The power level for the Hartman-Hahn condition on ${ }^{13} \mathrm{C}$ was found by varying the power level applied to the ${ }^{13} \mathrm{C}$ nuclei for a standard adamantane sample at a set contact time $(5 \mathrm{~ms})$ and locating the optimal value for maximal signal response. Power levels amounted to -3.5 and $0.32 \mathrm{~dB}$ for the ${ }^{1} \mathrm{H}$ and ${ }^{13} \mathrm{C}$ nuclei, respectively, utilizing 100 and 300 Watt amplifiers, respectively. Typically, a spectrum was acquired for $12 \mathrm{~h}$ consisting of 146 scans. $1 \mathrm{k}$ data points were collected for each FID (acquisition time $=6.4 \mathrm{~ms}$ ) which were processed by zero-filling to $2 \mathrm{k}$ and applying a line broadening of $100 \mathrm{~Hz}$. Selected samples were also rerun to gauge run-to-run aberrations, either sequentially for the same sample insertion, at different sessions for the same sample, or by repacking the sample rotor with the same material. For comparative purposes, spectra were vertically scaled and shifted horizontally. Solution-state spectra were acquired as previously described $[55,56]$.

\section{References}

[1] K. D. Klika, "Use of sub-stoichiometric amounts of chiral auxiliaries for enantiodifferentiation by NMR; caveats and potential utility," Tetrahedron: Asymmetry, vol. 20, no. 10, pp. 1099-1102, 2009.

[2] K. D. Klika, M. Budovská, and P. Kutschy, "Enantiodifferentiation of phytoalexin spirobrassinin derivatives using the chiral solvating agent (R)-(+)-1,1'-bi-2-naphthol in conjunction with molecular modeling," Tetrahedron: Asymmetry, vol. 21, no. 6, pp. 647-658, 2010.

[3] V. Nieminen, D. Yu. Murzin, and K. D. Klika, "NMR and molecular modeling of the dimeric self-association of the enantiomers of 1,1'-bi-2-napthol and 1-phenyl-2,2,2trifluoroethanol in the solution state and their relevance to enantiomer self-disproportionation on achiral-phase chromatography (ESDAC)," Organic \& Biomolecular Chemistry, vol. 7, no. 3, pp. 537-542, 2009.

[4] R. M. Carman and K. D. Klika, "The optical fractionation of a partially racemic natural product by chromatography over an achiral substrate," Australian Journal of Chemistry, vol. 44, no. 6, pp. 895-896, 1991.

[5] V. A. Soloshonok, "Remarkable amplification of the selfdisproportionation of enantiomers on achiral-phase chromatography columns," Angewandte Chemie International Edition, vol. 45, no. 5, pp. 766-769, 2006.

[6] R. Stephani and V. Cesare, "Enantiomeric enrichment of nonracemic antihistamines by achiral high-performance liquid chromatography," Journal of Chromatography A, vol. 813, no. 1, pp. 79-84, 1998.

[7] V. A. Soloshonok and D. O. Berbasov, "Self-disproportionation of enantiomers of (R)-ethyl 3-(3,5-dinitrobenzamido)-4,4,4trifluorobutanoate on achiral silica gel stationary phase," Journal of Fluorine Chemistry, vol. 127, no. 4-5, pp. 597-603, 2006.

[8] R. M. Carman and K. D. Klika, "Partially racemic compounds as brushtail possum urinary metabolites," Australian Journal of Chemistry, vol. 45, no. 4, pp. 651-657, 1992.

[9] R. M. Carman, A. C. Garner, and K. D. Klika, "2,9-dihydroxyand 2,10-dihydroxy-1,8-cineole. Two new possum urinary metabolites," Australian Journal of Chemistry, vol. 47, no. 8, pp. 1509-1521, 1994.

[10] V. A. Soloshonok and D. O. Berbasov, "Self-disproportionation of enantiomers on achiral phase chromatography. One more 
example of fluorine's magic powers," Chimica Oggi/Chemistry Today, vol. 24, no. 3, pp. 44-47, 2006.

[11] K. D. Klika, "Proposed extension to the natta projection notation system for enabling an indication of relative stereochemistry and the stereochemical state," International Journal of Organic Chemistry, vol. 1, no. 4, pp. 215-217, 2011.

[12] K. D. Klika, "Suggested new terms for describing chiral states and the state-dependent behavior of chiral systems," International Journal of Organic Chemistry. In press.

[13] D. Parker, "NMR determination of enantiomeric purity," Chemical Reviews, vol. 91, no. 7, pp. 1441-1457, 1991.

[14] J. M. Seco, E. Quiñoá, and R. Riguera, "The Assignment of Absolute Configuration by NMR,” Chemical Reviews, vol. 104, no. 1, pp. 17-117, 2004.

[15] J. M. Seco, E. Quiñoá, and R. Riguera, "A practical guide for the assignment of the absolute configuration of alcohols, amines and carboxylic acids by NMR," Tetrahedron: Asymmetry, vol. 12, no. 21, pp. 2915-2925, 2001.

[16] F. Freire, J. M. Seco, E. Quiñoá, and R. Riguera, “Determining the absolute stereochemistry of secondary/secondary diols by 1H NMR: basis and applications," Journal of Organic Chemistry, vol. 70, no. 10, pp. 3778-3790, 2005.

[17] A. F. Casey, "Chiral discrimination by NMR spectroscopy," TrAC Trends in Analytical Chemistry, vol. 12, no. 4, pp. 185-189, 1993.

[18] E. Tadeusiak, "Studies of racemate/enantiomer systems and determination of enantiomeric excess by high-resolution liquid and solid-state NMR spectroscopy," Wiadomości Chemiczne, vol. 57, no. 5-6, pp. 367-389, 2003.

[19] R. Rothchild, "NMR methods for determination of enantiomeric excess," Enantiomer, vol. 5, no. 5, pp. 457-471, 2000.

[20] W. Lee, E. Bang, C. S. Baek, and W. Lee, "Chiral discrimination studies of (+)-(18-crown-6)-2,3,11,12-tetracarboxylic acid by high-performance liquid chromatography and NMR spectroscopy," Magnetic Resonance in Chemistry, vol. 42, no. 4, pp. 389-395, 2004.

[21] Y.-S. Zheng and C. Zhang, "Exceptional chiral recognition of racemic carboxylic acids by calix[4]arenes bearing optically pure $\alpha, \beta$-amino alcohol groups," Organic Letters, vol. 6, no. 8, pp. 1189-1192, 2004.

[22] J. M. Rivera, T. Martín, and J. Rebek Jr., "Chiral spaces: dissymmetric capsules through self-assembly," Science, vol. 279, no. 5353, pp. 1021-1023, 1998.

[23] D. Fiedler, D. H. Leung, R. G. Bergman, and K. N. Raymond, "Enantioselective guest binding and dynamic resolution of cationic ruthenium complexes by a chiral metal-ligand assembly," Journal of the American Chemical Society, vol. 126, no. 12, pp. 3674-3675, 2004.

[24] Y. Liu, E. C. Yang, Y. W. Yang et al., "Thermodynamics of the molecular and chiral recognition of cycloalkanols and camphor by modified beta-cyclodextrins possessing simple aromatic tethers," Journal of Organic Chemistry, vol. 69, no. 1, pp. 173-180, 2004

[25] J. M. Péchiné, A. Meddour, and J. Courtieu, "Monitoring the differential ordering of enantiomers included into cyclodextrins through deuterium NMR in lyotropic liquid crystals," Chemical Communications, no. 16, pp. 1734-1735, 2002.

[26] T. J. Wenzel and J. D. Wilcox, "Chiral reagents for the determination of enantiomeric excess and absolute configuration using NMR spectroscopy," Chirality, vol. 15, no. 3, pp. 256-270, 2003.
[27] P. Lesot, M. Sarfati, and J. Courtieu, "Natural abundance deuterium NMR spectroscopy in polypeptide liquid crystals as a new and incisive means for the enantiodifferentiation of chiral hydrocarbons," Chemistry, vol. 9, no. 8, pp. 1724-1745, 2003.

[28] M. Sarfati, P. Lesot, D. Merlet, and J. Courtieu, “Theoretical and experimental aspects of enantiomeric differentiation using natural abundance multinuclear nmr spectroscopy in chiral polypeptide liquid crystals," Chemical Communications, no. 21, pp. 2069-2081, 2000.

[29] C. Aroulanda, V. Boucard, F. Guibé, J. Courtieu, and D. Merlet, "Weakly oriented liquid-crystal NMR solvents as a general tool to determine relative configurations," Chemistry, vol. 9, no. 18, pp. 4536-4539, 2003.

[30] A. Solgadi, A. Meddour, and J. Courtieu, "Enantiomeric discrimination of water soluble compounds using deuterium NMR in a glucopon/buffered water/n-hexanol chiral lyotropic liquid crystal," Tetrahedron: Asymmetry, vol. 15, no. 8, pp. 1315-1318, 2004.

[31] M. Tavasli, J. Courtieu, R. J. Goss, A. Meddour, and D. O’Hagan, "Extreme enantiomeric discrimination of fluoroalkanes using deuterium NMR in chiral liquid crystalline media," Chemical Communications (Cambridge, England), no. 8, pp. 844-845, 2002.

[32] M. Sarfati, J. Courtieu, and P. Lesot, "First successful enantiomeric discrimination of chiral alkanes using NMR spectroscopy," Chemical Communications, no. 13, pp. 1113-1114, 2000.

[33] V. Madiot, P. Lesot, D. Grée, J. Courtieu, and R. Gree, "Highly enantioselective propargylic monofluorination established by carbon-13 and fluorine-19 NMR in chiral liquid crystals," Chemical Communications, no. 2, pp. 169-170, 2000.

[34] C. Aroulanda, D. Merlet, J. Courtieu, and P. Lesot, "NMR experimental evidence of the differentiation of enantiotopic directions in $\mathrm{Cs}$ and $\mathrm{C} 2 \mathrm{v}$ molecules using partially oriented, chiral media," Journal of the American Chemical Society, vol. 123, no. 48, pp. 12059-12066, 2001.

[35] J. Farjon, D. Merlet, P. Lesot, and J. Courtieu, "Enantiomeric excess measurements in weakly oriented chiral liquid crystal solvents through 2D $1 \mathrm{H}$ selective refocusing experiments," Journal of Magnetic Resonance, vol. 158, no. 1-2, pp. 169-172, 2002.

[36] P. Lesot, O. Lafon, J. Courtieu, and P. Berdagué, "Analysis of the ${ }^{13} \mathrm{C}$ NMR spectra of molecules, chiral by isotopic substitution, dissolved in a chiral oriented environment: towards the absolute assignment of the pro-R/pro-S character of enantiotopic ligands in prochiral molecules," Chemistry, vol. 10, no. 15, pp. 3741-3746, 2004.

[37] P. Lesot, D. Merlet, M. Sarfati, J. Courtieu, H. Zimmermann, and Z. Luz, "Enantiomeric and enantiotopic analysis of coneshaped compounds with C3 and C3v symmetry using NMR spectroscopy in chiral anisotropic solvents," Journal of the American Chemical Society, vol. 124, no. 34, pp. 10071-10082, 2002.

[38] J. Farjon, J. P. Baltaze, P. Lesot, D. Merlet, and J. Courtieu, "Heteronuclear selective refocusing 2D NMR experiments for the spectral analysis of enantiomers in chiral oriented solvents," Magnetic Resonance in Chemistry, vol. 42, no. 7, pp. 594-599, 2004.

[39] E. Sackmann, S. Meiboom, and L. C. Snyder, "The nuclear magnetic resonance spectra of enantiomers in optically active liquid crystals," Journal of the American Chemical Society, vol. 90, no. 8, pp. 2183-2184, 1968. 
[40] E. Sackmann, S. Meiboom, L. C. Snyder, A. E. Meixner, and R. E. Dietz, "On the structure of the liquid crystalline state of cholesterol derivatives," Journal of the American Chemical Society, vol. 90, no. 13, pp. 3567-3569, 1968.

[41] K. Kobzar, H. Kessler, and B. Luy, "Stretched gelatin gels as chiral alignment media for the discrimination of enantiomers by NMR spectroscopy," Angewandte Chemie-International Edition, vol. 44, no. 20, pp. 3145-3147, 2005.

[42] U. Eliav and G. Navon, "Collagen fibers as a chiral agent: a demonstration of stereochemistry effects," Journal of the American Chemical Society, vol. 128, no. 50, pp. 15956-15957, 2006.

[43] A. Tsourkas, O. Hofstetter, H. Hofstetter, R. Weissleder, and L. Josephson, "Magnetic relaxation switch immunosensors detect enantiomeric impurities," Angewandte Chemie-International Edition, vol. 43, no. 18, pp. 2395-2399, 2004.

[44] J. Crassous, C. Chardonnet, T. Saue, and P. Schwerdtfeger, "Recent experimental and theoretical developments towards the observation of parity violation (PV) effects in molecules by spectroscopy," Organic \& Biomolecular Chemistry, vol. 3, no. 12, pp. 2218-2224, 2005.

[45] H. D. W. Hill, A. P. Zens, and J. Jacobus, "Solid-state NMR spectroscopy. Distinction of diastereomers and determination of optical purity," Journal of the American Chemical Society, vol. 101, no. 23, pp. 7090-7091, 1979.

[46] K. V. Andersen, H. Bildsoe, and H. Jakobsen, “Determination of enantiomeric purity from solid-state 31P MAS NMR of organophosphorus compounds," Magnetic Resonance in Chemistry, vol. 28, no. 13, pp. S47-S51, 1990.

[47] H. Tsuji, F. Horii, M. Nakagawa, Y. Ikada, H. Odani, and R. Kitamaru, "Stereocomplex formation between enantiomeric poly(lactic acid)s. 7. Phase structure of the stereocomplex crystallized from a dilute acetonitrile solution as studied by highresolution solid-state ${ }^{13} \mathrm{C}$ NMR spectroscopy," Macromolecules, vol. 25, no. 16, pp. 4114-4118, 1992.

[48] P. Tekely, C. Gardiennet, M. J. Potrzebowski, A. Sebald, D. Reichert, and Z. Luz, "Probing molecular geometry of solids by nuclear magnetic resonance spin exchange at the $n=0$ rotational-resonance condition," Journal of Chemical Physics, vol. 116, no. 17, pp. 7607-7616, 2002.

[49] M. J. Potrzebowski, E. Tadeusiak, K. Misiura, W. Ciesielski, G. Bujacz, and P. Tekely, "A new method for distinguishing between enantiomers and racemates and assignment of enantiomeric purity by means of solid-state NMR. Examples from oxazaphosphorinanes," Chemistry, vol. 8, no. 21, pp. 5007-5011, 2002.

[50] M. J. Potrzebowski, "What high-resolution solid-state NMR spectroscopy can offer to organic chemists," European Journal of Organic Chemistry, vol. 2003, no. 8, pp. 1367-1376, 2003.

[51] M. J. Potrzebowski, A. Jeziorna, and S. Kaźmierski, "NMR studies of chiral organic compounds in non-isotropic phases," Concepts in Magnetic Resonance Part A, vol. 32, no. 3, pp. 201-218, 2008.

[52] S. Nakamura, F. Imashiro, K. Takegoshi, and T. Terao, "Sequential arrangement of $\gamma$-vaierolactone enantiomers enclathrated in cholic acid channels as studied by ${ }^{13} \mathrm{C}$ solid-state NMR: elucidation of the optical resolution mechanism," Journal of the American Chemical Society, vol. 126, no. 28, pp. 8769-8776, 2004.
[53] A. B. Ouryupin, M. I. Kadyko, P. V. Petrovskii et al., "Enantiomeric 2-anilino-2-oxo-1,3,2-oxazaphosphorinanes: synthesis and NMR-investigation of their non-racemic mixtures," Tetrahedron: Asymmetry, vol. 6, no. 7, pp. 1813-1824, 1995.

[54] F. Toda, K. Tanaka, H. Miyamoto, H. Koshima, I. Miyahara, and K. Hirotsu, "Formation of racemic compound crystals by mixing of two enantiomeric crystals in the solid state. Liquid transport of molecules from crystal to crystal," Journal of the Chemical Society. Perkin Transactions 2, no. 9, pp. 1877-1885, 1997.

[55] J. Mäki, P. Tähtinen, L. Kronberg, and K. D. Klika, "Restricted rotation/tautomeric equilibrium and determination of the site and extent of protonation in bi-imidazole nucleosides by multinuclear NMR and GIAO-DFT calculations," Journal of Physical Organic Chemistry, vol. 18, no. 3, pp. 240-249, 2005.

[56] P. Virta, A. Koch, M. U. Roslund et al., "Synthesis, characterisation and theoretical calculations of 2,6-diaminopurine etheno derivatives," Organic \& Biomolecular Chemistry, vol. 3, no. 16, pp. 2924-2929, 2005. 

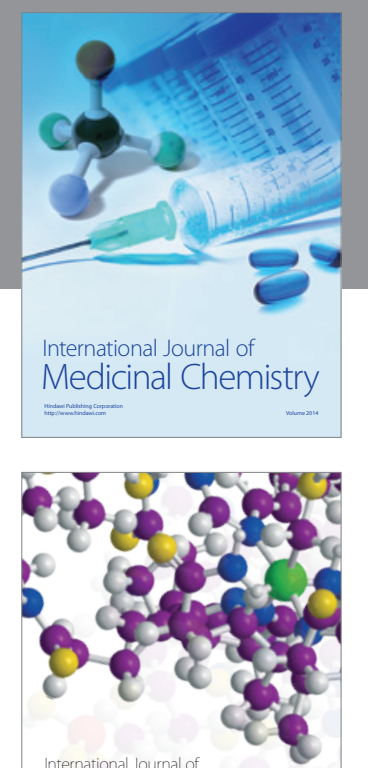

\section{Carbohydrate} Chemistry

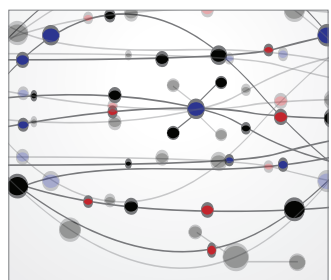

The Scientific World Journal
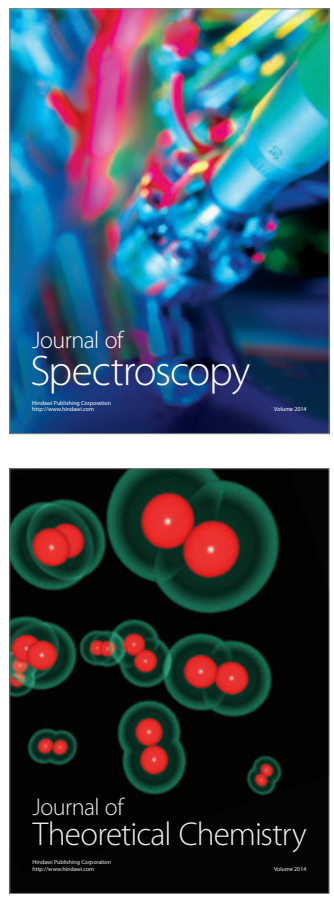
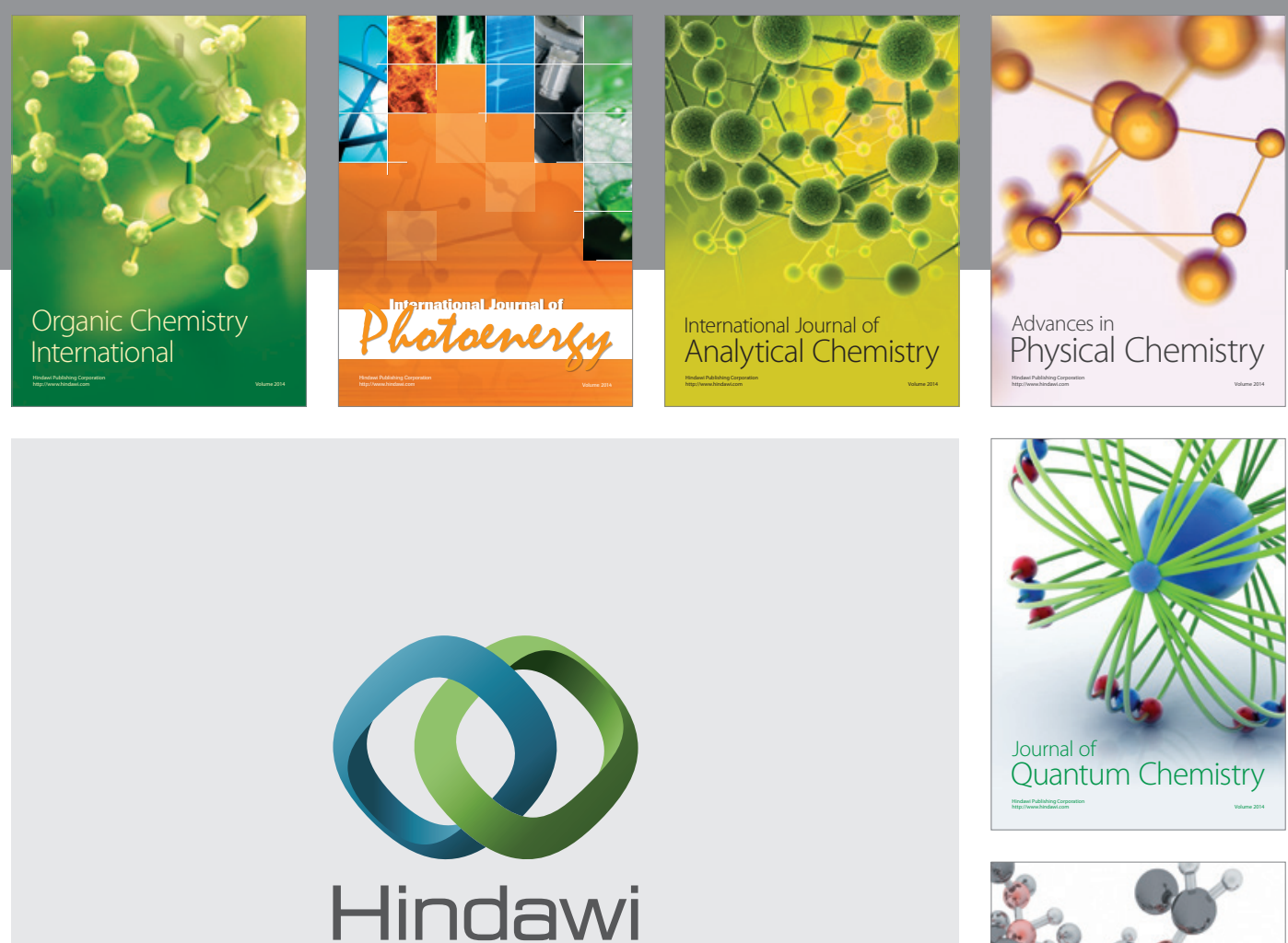

Submit your manuscripts at

http://www.hindawi.com

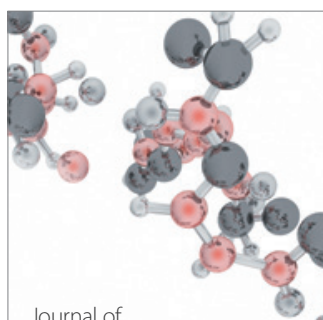

Analytical Methods

in Chemistry

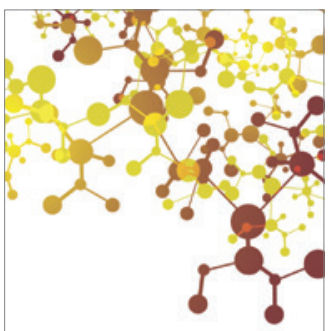

Journal of

Applied Chemistry

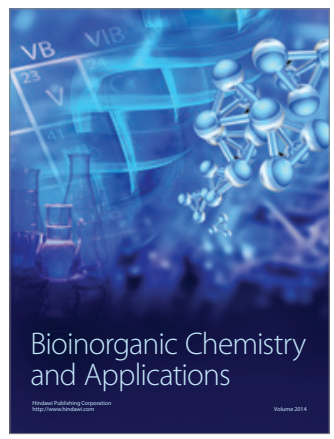

Inorganic Chemistry
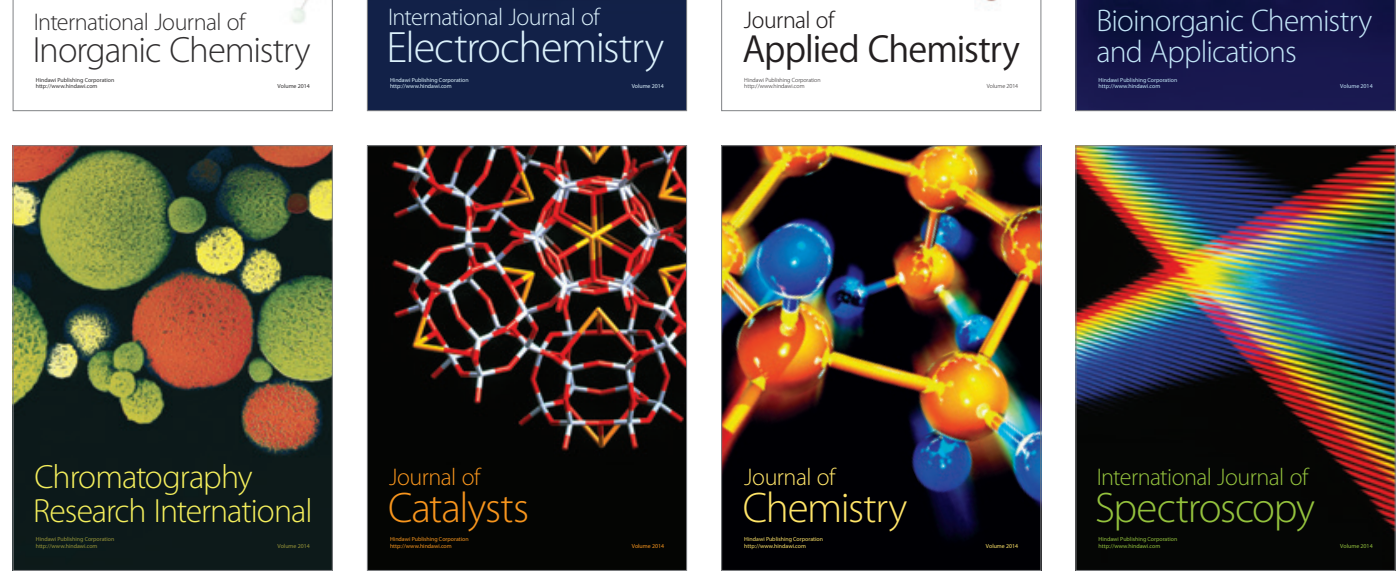